\title{
Ist Diabetes heilbar durch bariatrische Chirurgie? - \\ Mechanismen der Verbesserung der diabetischen \\ Stoffwechsellage durch chirurgische Adipositastherapie -
}

\section{Is it possible to cure diabetes mellitus with bariatric surgery? - Mechanisms of improvement of diabetes by bariatric surgery -}

Autor

Matthias Weck

Institut

Medizinische Klinik 3, HELIOS Weißeritztal-Kliniken, Freital, Germany

Schlüsselwörter

Diabetes mellitus Typ 2, Adipositas, bariatrische Chirurgie

Key words

diabetes mellitus type 2 , obesity, bariatric surgery

eingereicht 09.06 .2017

akzeptiert 29.08.2017

Bibliografie

DOI https://doi.org/10.1055/s-0043-119075

Online-Publikation: 20.9.2017

Diabetologie 2017; 12: 372-385

(c) Georg Thieme Verlag KG, Stuttgart · New York

ISSN 1861-9002

Korrespondenzadresse

Prof. Matthias Weck

Medizinische Klinik 3, HELIOS Weißeritztal-Kliniken,

Bürgerstr. 7, 01705 Freital, Germany

Tel.: ++49/3 51/6466916

Fax: $++49 / 351 / 6467067$

Matthias.Weck@helios-kliniken.de

\section{ZUSAMMENFASSUNG}

In den letzten Jahren wurden die Ergebnisse randomisierter kontrollierter Studien publiziert, die im 5-Jahres-Verlauf die metabolischen Effekte der bariatrischen Chirurgie mit konventionellen Formen der Gewichtsreduktion vergleichen. Diese Studien zeigen unisono, dass die bariatrische Chirurgie hinsichtlich der Besserung der diabetischen Stoffwechsellage den konventionellen Behandlungsformen signifikant überlegen ist. Die Diabetesremissionsraten variieren abhängig von Ausgangsparametern, Operationsmethode und Follow-upDauer zwischen 95 und $23 \%$.

Ist Diabetes heilbar durch bariatrische Chirurgie? Die klare Antwort muss lauten: Nein, aber
1. $35-50 \%$ der Patienten mit Typ-2-Diabetes, die mittels bariatrischer Chirurgie initial nach Operation eine Diabetesremission erreichen konnten, erleben im weiteren Verlauf das Wiederkehren der „Krankheit“ d. h. erneut ansteigende Blutzuckerwerte.

2. Die mittlere „krankheitsfreie Zeit“ beträgt 8,3 Jahre (Range 5-15 Jahre)

3. Jedes Jahr, das die Patienten mit Typ-2-Diabetes in der Remission der Erkrankung verbrachten, war mit einer Reduktion des Risikos mikrovaskulärer Komplikationen um 19\% verbunden.

Insofern ist die bariatrische Chirurgie in Form von Roux-en-Y Gastric Bypass (RYGB), Laparoscopic Sleeve Gastrectomy (LSG) und den neueren Verfahren wie Omega Loop Bypass („Mini-Bypass“) oder biliopankreatische Diversion (BPD) eine wirkungsvolle therapeutische Option in der Behandlung des Diabetes mellitus Typ 2 und verringert offenbar auch das Risiko des Auftretens von mikrovaskulären Diabetesfolgekomplikationen. Je früher im Krankheitsverlauf die bariatrische Chirurgie SSherangezogen wird, desto effektiver scheinen diese Verfahren zu sein.

Welche der Operationen für Patienten mit Typ-2-Diabetes am besten geeignet ist, ist derzeit nicht definitiv entschieden. Der RYGB scheint etwas effektiver zu sein. Die Verfahren der bariatrischen Chirurgie gehören in das Spektrum der differenzialtherapeutischen Überlegungen insbesondere bei adipösen Patienten mit Typ-2-Diabetes mit einem BMI > $35 \mathrm{~kg} / \mathrm{m}^{2}$.

Die Mechanismen der Verbesserung der diabetischen Stoffwechsellage durch bariatrische Operationen werden anhand der aktuellen Literatur detailliert beschrieben. Die Indikationen, Kontraindikationen, Komplikationen und Therapiealgorithmen der bariatrischen Chirurgie bei Typ-2-Diabetes sind in den entsprechenden Leitlinien ausführlich dargestellt und nicht Gegenstand dieser Publikation.

\section{ABSTRACT}

Recently, the results of long-term randomized controlled studies were published comparing the metabolic effects of bariatric surgery with conventional treatment strategies of obesity. Unanimously, these studies demonstrate that baria- 
tric surgery improves glycemic control significantly compared to conventional medical treatment. Rates of diabetes remission range from 95 to $23 \%$ depending on initial patients characteristics, operation method of bariatric surgery and followup duration.

Is diabetes curable with bariatric surgery? The clear answer is: no, but

1. $35-50 \%$ of diabetic patients, achieving diabetes remission after bariatric surgery, had a recurrence of the disease in terms of increasing blood glucose values during the following years.

2. The mean "diabetes-free" time was 8.3 years (range $5-15$ years).

3. Each year that diabetic patients were in the remission of the disease, was associated with a reduction of the risk of microvascular complications of $19 \%$.

Therefore, bariatric surgery with Roux-en-Y gastric bypass (RYGB), laparoscopic sleeve gastrectomy (LSG), biliopancrea- tic diversion (BPD) or newer techniques as omega loop bypass are powerful therapeutic options in the treatment of diabetes and obesity. Bariatric surgery seems to reduce the risk of microvascular diabetic complications. Therefore, bariatric surgery should be involved in the therapeutic strategies as early as possible.

It is not definitively clear which type of bariatric operation is the best option for diabetic patients. RYGB seems to have some benefits. The methods of bariatric surgery should be included into the therapeutic armamentarium of each diabetes specialist.

The mechanisms of improvement of glycemic control by bariatric surgery will be described in detail according to recent literature data.

Indications, contraindications, complications or therapeutic algorithms of bariatric surgery of type 2 diabetes are listed in detail at common guidelines and are not part of this review.

\section{Einleitung}

Die enge Assoziation zwischen Adipositas und Diabetes mellitus Typ 2 ist bestens belegt. Gewichtsreduktion bessert in aller Regel die diabetische Stoffwechsellage dramatisch. Allerdings belegt eine Vielzahl von Studien, dass fast alle Probanden, die durch energiereduzierte Ernährung, gesteigerte körperliche Aktivität in Kombination mit Verhaltenstherapie (sog. multimodaler Therapieansatz) Gewicht verloren haben, ihr Ausgangsgewicht innerhalb von 5 Jahren wieder erreicht bzw. sogar überschritten haben [1]. Dies wird gern als Jo-Jo-Effekt bezeichnet.

Pories beschrieb 1992 erstmals den Zusammenhang zwischen einer gewichtsreduzierenden Operation und einer deutlichen Verbesserung der diabetischen Stoffwechsellage [2]. Christou et al. [3] konnten exzellente Ergebnisse der bariatrischen Chirurgie bei einer Vielzahl von Erkrankungsformen an über 1000 operierten Patienten nachweisen. Die SOS-Studie zeigte im Verlauf von $>15$ Jahren nach bariatrischer Operation eine deutliche Reduktion der Diabetesinzidenz und sehr gute Raten an Diabetesremission $[4,5]$. Inzwischen liegen auch die Daten von mehreren randomisierten kontrollierten Studien vor, die u. a. die metabolischen Effekte der bariatrischen Chirurgie mit konventionellen Formen der Gewichtsreduktion vergleichen [6 - 12]. Diese Studien zeigen unisono, dass die bariatrische Chirurgie hinsichtlich der Besserung der diabetischen Stoffwechsellage den konventionellen Behandlungsformen signifikant überlegen ist. Dies führte manche Kollegen zu der Auffassung, dass Diabetes mittels bariatrischer Chirurgie „heilbar“ sei.

Im Folgenden wird beschrieben, welche Mechanismen zur dramatischen Besserung der diabetischen Stoffwechsellage im Gefolge der bariatrischen Chirurgie beitragen. Basis dieser Darstellung sind die nach Auffassung des Autors wesentlichen Studien zur bariatrischen Chirurgie bei Diabetes mellitus.

\section{Remission des Diabetes}

Die ADA hat 2009 Kriterien definiert, die eine Remission des Diabetes annehmen lassen, insbesondere um Daten aus verschiedenen Studien besser vergleichbar zu machen. Das ADA-Statement ist überschrieben mit: „How do we define cure of diabetes?“. Diese Überschrift lässt „Heilung“ vermuten, Remission dürfte gemeint sein [13].

komplette Remission:

- NBZ<5,6 mmol/l

- HbA1c $<6,0 \%$

- frei von Antidiabetika seit mindestens 1 Jahr

partielle Remission:

- NBZ<5,6 - 7,0 mmol/l

- HbA1c <6,5\%

- frei von Antidiabetika seit mindestens 1 Jahr

\section{Wesentliche Studien zu den Effekten der bariatrischen Chirurgie bei Diabetes mellitus Typ 2}

\section{Christou-Studie}

Eine eher wenig beachtete Publikation zum Effekt des Magenbypass auf Morbidität und Mortalität verschiedener Erkrankungen im Vergleich zu einer konventionell behandelten Kontrollgruppe stammt von Christou et al. aus dem Jahre 2004 [3]. Es wurden 1035 Patienten hauptsächlich einem Magenbypass unterzogen und eine relative Reduktion der Gesamtmortalität um $89 \%$ ( $\mathrm{Cl} 0,04-0,27)$ bei einer relativen Risikoreduktion an Diabetes mellitus von $65 \%$ beschrieben ( $\triangleright$ Tab. 1 ).

Es handelt sich einschränkend um eine nicht randomisierte kontrollierte Studie. 
- Tab. 1 Reduktion von Morbidität und Mortalität nach bariatrischer Chirurgie in der Studie von Christou et al. (modifiziert nach [3]). Fünfjahresmorbidität und Mortalität.

\begin{tabular}{|c|c|c|c|c|c|c|}
\hline \multirow[b]{3}{*}{ Erkrankungen } & \multicolumn{4}{|c|}{ Kohorte } & \multirow{3}{*}{$\begin{array}{l}\text { Relative Risiko- } \\
\text { reduktion } \\
\%\end{array}$} & \multirow[t]{3}{*}{$\mathbf{p}$} \\
\hline & \multicolumn{2}{|c|}{ Adipositas-Chirurgie $(n=1035)$} & \multicolumn{2}{|c|}{$\begin{array}{l}\text { Kontrollen } \\
(n=5746)\end{array}$} & & \\
\hline & $\mathbf{n}$ & $\%$ & $\mathbf{n}$ & $\%$ & & \\
\hline Blut & 4 & 0,39 & 41 & 0,72 & 46 & 0,230 \\
\hline Krebs & 21 & 2,03 & 487 & 8,49 & 76 & 0,001 \\
\hline Kardiovaskuläre & 49 & 4,73 & 1530 & 26,69 & 82 & 0,001 \\
\hline Magen-Darm & 377 & 36,43 & 1414 & 24,66 & +48 & 0,001 \\
\hline Endokrine & 98 & 9,47 & 1566 & 27,25 & 65 & 0,001 \\
\hline Urologische & 77 & 7,44 & 551 & 9,61 & 23 & 0,027 \\
\hline Infektionen & 90 & 8,70 & 2140 & 37,33 & 77 & 0,001 \\
\hline Muskel/Skelett & 50 & 4,83 & 682 & 11,90 & 59 & 0,001 \\
\hline Nervensystem & 25 & 2,42 & 228 & 3,98 & 39 & 0,01 \\
\hline Psychiatrie & 45 & 4,35 & 470 & 8,20 & 47 & 0,001 \\
\hline Respiration & 28 & 2,71 & 651 & 11,36 & 76 & 0,001 \\
\hline Haut & 38 & 3,67 & 305 & 5,32 & 31 & 0,027 \\
\hline Mortalität & 7 & 0,68 & 354 & 6,17 & 89 & 0,001 \\
\hline
\end{tabular}

\section{SOS-Studie}

Die SOS-Studie, in deren Rahmen ca. 2000 Probanden einer bariatrischen Therapie und 2000 Probanden einer konventionellen Gewichtsreduktion unterzogen wurden, zeigte folgende Resultate zugunsten der bariatrischen Chirurgie, wobei der Anteil an Patienten mit Diabetes zu Beginn der Studie gering war:

- eine Reduktion der Gesamtmortalität um 30\% nach 15 Jahren [14]

- Remission des Diabetes in $72 \%$ der Probanden nach 2 Jahren, nach 10 Jahren noch in $36 \%[15,16]$

- Inzidenz an Diabetes bei $1 \%$ der Probanden nach 2 Jahren bzw. $7 \%$ nach 10 Jahren (Kontrollgruppe 8 vs. 24\%) [15 - 18]

- signifikante Reduktion kardiovaskulärer Ereignisse in der operierten Gruppe der Diabetiker der SOS-Studie nach 20 Jahren (alle kardiovaskulären Ereignisse, Herzinfarkt) [4, 5] (

\section{Mingrone-Studie}

In einer Single-Center-Studie, nicht verblindet, aber randomisiertkontrolliert, wurden von G. Mingrone und Mitarbeitern [9, 10] 60 Patienten im Alter von $30-60$ Jahren, BMI $>35 \mathrm{~kg} / \mathrm{m}^{2}$, mindestens 5 Jahre Diabetesdauer und einen $\mathrm{HbA} 1 \mathrm{c}>7 \%$ über bisher 5 Jahre untersucht. Die Probanden wurden randomisiert 1:1:1 einer konventionellen medizinischen Therapie, einem Roux-Y-Magenbypass (RYGB) oder der biliopankreatischen Diversion (BPD) unterzogen, d. h. 20 Patienten pro Gruppe. Primärer Endpunkt war die Diabetesremission nach 2 Jahren. Kriterien für eine Remission waren $\mathrm{NBZ}<5,6 \mathrm{mmol} / \mathrm{l}, \mathrm{HbA} 1 \mathrm{c}<6,5 \%$, keine antidiabetische Medikation. Abb. 2 zeigt die Basisdaten der Probanden und den Verlauf des Körpergewichts und des HbA1c in den drei Gruppen.
Nach zwei Jahren zeigt sich eine Diabetesremission von 75\% beim Magenbypass und von $95 \%$ bei der BPD.

In 2015 wurden die 5-Jahres-Daten dieser Studie veröffentlicht [10]. $88 \%$ der Probanden beendeten den 5-Jahres-Follow-up. Die Diabetesremission blieb bestehen bei $37 \%$ der Probanden mit RYGB und bei $63 \%$ der BPD-Patienten. Keiner der Probanden in der konventionellen Therapiegruppe hatte eine Diabetesremission nach 5 Jahren. Das Ausmaß der Gewichtsreduktion hatte keinen prädiktiven Wert hinsichtlich der Diabetesremission. Dies trotz einer durchschnittlichen Gewichtsreduktion um $37 \mathrm{~kg}$ bei RYGB bzw. 44,7 kg bei BPD. Die Patienten der konventionell behandelten Gruppe konnten ihr Gewicht im Schnitt um $10 \mathrm{~kg}$ in den 5 Jahren Follow-up reduzieren. Ein Hyperglykämie-Rezidiv trat bei $44 \%$ der Probanden der RYGB- und der BPD-Gruppe auf, die nach 2 Jahren in Remission waren, allerdings bei einem mittleren HbA1c von $6,7 \%$ und entweder unter Diät allein oder in Kombination mit Metformin. Generell kann eine deutliche Verbesserung der Glykämie unter bariatrischer Gewichtsreduktion mittels RYGB oder BPD konstatiert werden. $82 \%$ der operierten Probanden hatten nach 5 Jahren einen $\mathrm{HbA} 1 \mathrm{c}<7,0 \%$.

Surrogatparameter des kardiovaskulären Risikos (HbA1c, LDLCholesterol, systolischer und diastolischer Blutdruck) fielen in allen 3 Studiengruppen deutlich ab. Das daraus abgeleitete kardiovaskuläre Risiko war nach 5 Jahren in den beiden bariatrischen Gruppen halb so hoch wie bei den Kontrollen. Ebenso war der Bedarf an kardiovaskulären Medikamenten in den bariatrischen Gruppen deutlich niedriger. Diabeteskomplikationen finden sich bei RYGB und BPD bis auf wenige Ausnahmen im 5-JahresFollow-up nicht. Natürlich ist die Fallzahl (und die Beobachtungs- 


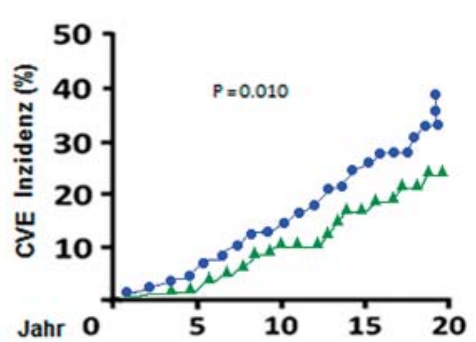

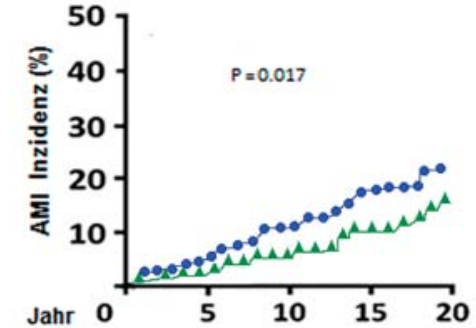

$\bullet$ kontrollen

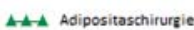

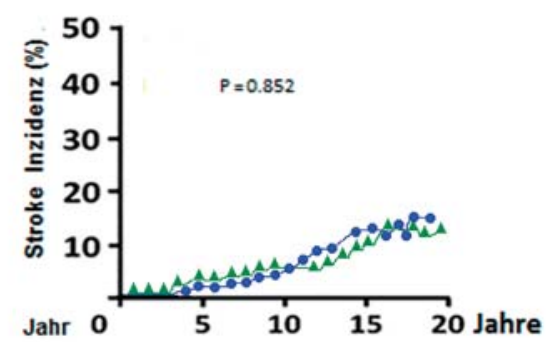

CVE ........cardiovaskuläre Ereignisse AMI...... akuter Myokardinfarkt NNT für $\mathrm{AMI}=16$

- Abb. 1 Kumulative Inzidenz kardiovaskulärer Ereignisse bei Typ-2-Diabetikern in der SOS-Studie (nach [5]). CVE cardiovaskuläre Ereignisse. AMI akuter Myokardinfarkt. NNT für AMI = 16

dauer) für eine valide Aussage diesbezüglich zu gering, aber diese Schlussfolgerungen werden u. a. durch die SOS-Studie gestützt.

\section{STAMPEDE (Surgical Therapy and Medications Poten- tially Eradicate Diabetes Efficiently) Studie}

Auch von der Gruppe um P. Schauer [6 - 8] wurde eine randomisierte, nicht verblindete, Single-Center-Studie vorgelegt, ebenfalls im Design 1:1:1 mit jeweils 50 Patienten unter konventioneller Therapie, Magenbypass und laparoskopischer Sleeve-Gastrektomie (LSG). Der BMI dieser Patienten war im Vergleich zu Mingrone et al. [9] deutlich niedriger mit ca. 1/3 der Probanden mit einem $\mathrm{BMI}<35 \mathrm{~kg} / \mathrm{m}^{2}$.

Nach 12 Monaten wiesen die operierten Patienten eine Reduktion des BMI um 9- 10 Punkte auf, der HbA1c sank um 3\%. 42\% der Probanden nach Magenbypass und 37 \% nach LSG wiesen eine Remission des Diabetes nach 1 Jahr auf. Dabei galt ein HbA1c $<6 \%$ als Remission. HbA1c $<6 \%$ und keinerlei antidiabetische Medikation hatten $42 \%$ der Probanden nach RYGB und $27 \%$ der Patienten nach LSG. Insgesamt also ein Trend zu günstigeren Diabetesparametern nach Magenbypass, der allerdings statistisch nicht signifikant war ( $\mathbf{A b b}$. 3).

2017 wurden die 5-Jahres-Ergebnisse dieser Studie publiziert [8]. Die Kriterien für die Diabetesremission erreichten nach 5 Jahren $5 \%$ der Patienten unter konventioneller Therapie, $29 \%$ unter Magenbypass und $23 \%$ unter LSG. Die Unterschiede waren nur unadjustiert signifikant. Patienten unter RYGB und LSG hatten aber signifikant bessere Chancen, einen HbA1c $\leq 6 \%$ mit oder ohne Antidiabetika zu erzielen. Auch die Messwerte der Lebensqualität besserten sich in beiden bariatrischen Gruppen im Vergleich zu den konventionell behandelten Patienten. Nach 5 Jahren hatten Patienten unter RYGB einen größeren Gewichtsverlust und weniger Diabetesmedikamente als Patienten unter LSG, wobei diese Unterscheide nicht signifikant waren. Die Frage, welche Operationsmethode für Diabetiker „besser“ ist, lässt sich also weiterhin nicht beantworten. Eine wichtige Aussage der STAMPEDE-Studie dürfte sein, dass Patienten mit einem BMI von
$27-34 \mathrm{~kg} / \mathrm{m}^{2}$ eine vergleichbare Besserung der Glykämie erreichen konnten wie Patienten mit einem BMI > $35 \mathrm{~kg} / \mathrm{m}^{2}$ ( $\triangleright$ Abb. 2).

\section{CROSSROADS (Calorie Reduction Or Surgery: Seeking to Reduce Obesity And Diabetes Study)}

Von Cummings et al. [11] wird nun erstmals eine „echte“ RCT vorgestellt mit dem Ziel, die Effekte des laparoskopischen Magenbypass (RYGB) mit denen einer intensivierten Lebensstil- und medikamentösen Intervention (ILMI) zu vergleichen. Bereits die Rekrutierung der Probanden zeigt das Dilemma solcher Studien. Von 1808 zum Screening eingeladenen Patienten verbleiben letztlich 43 Probanden zur PC-generierten Randomisierung 1:1 in RYGB oder ILMI. 23 Probanden wurden RYGB und 20 ILMI zugeordnet. 11 Probanden entzogen danach ihre Zustimmung, so dass 15 RYGB und 17 ILMI verblieben.

Als primärer Endpunkt galt eine Diabetesremission nach 1 Jahr (HbA1c $<6 \%$ und keine Antidiabetika). Die beiden Gruppen waren gut vergleichbar bei allerdings signifikant längerer Diabetesdauer in der RYGB-Gruppe (11,4 $\pm 4,8$ vs. 6,8 \pm 5,2 Jahre). Die BMI-Ausgangsdaten liegen mit 38,3 $\pm 3,7$ (RYGB) vs. 37,1 $\pm 3,5$ (ILMI) nicht im Bereich der Adipositas Grad III ( $\triangleright$ Abb. 4).

Die Diabetesremissionsraten nach den gewählten Kriterien waren $60 \%$ unter RYGB und 5,9\% mittels ILMI. Der HbA1c-Abfall war in beiden Gruppen eher moderat: von 7,7 $\pm 1,0 \%$ auf $6,4 \pm 1,6 \%$ unter RYGB und von 7,3 $\pm 0,9 \%$ auf $6,9 \pm 1,3 \%$ unter ILMI. Aus unserer Sicht ist CROSSROADS infolge der derzeit noch geringen Follow-up-Dauer und der ja ohnehin geringen Probandenzahl nicht geeignet, generalisierbare Schlussfolgerungen zu ziehen. Die generell unter bariatrischen Operationen zu verzeichnenden positiven Effekte auf den Glukosestoffwechsel und kardiovaskuläre Risikofaktoren zeigt auch diese Studie, ganz besonders, da die Maßnahmen der ILMI extrem aufwendig gestaltet waren. 


\section{A Anthropometrische Daten}

Basisdaten der Patienten

\begin{tabular}{|l|l|l|l|l|}
\hline & $\begin{array}{l}\text { Konventionelle } \\
\text { Gewichtsreduktion } \\
(\mathbf{n = 2 0 )}\end{array}$ & $\begin{array}{l}\text { RYGB } \\
(\mathbf{n = 2 0 )}\end{array}$ & $\begin{array}{l}\text { BPD } \\
(\mathbf{n = 2 0})\end{array}$ \\
\hline Alter & 43.5 & 43.9 & 42.8 & n.s. \\
\hline NBG $(\mathrm{mmol} / \mathrm{l})$ & 9.9 & 9.6 & 9.7 & n.s. \\
\hline HbA1C & 8.5 & 8.6 & 8.9 & n.s. \\
\hline BMI $\left(\mathrm{kg} / \mathrm{m}^{2}\right)$ & 45.6 & 44.8 & 45.1 & n.s. \\
\hline Diabetesdauer $(\mathrm{J})$ & 6.1 & 6.0 & 6.0 & n.s. \\
\hline
\end{tabular}

NBG: Nüchtern-Blutglucose.

B Verlauf von Körpergewicht und HbA1c über 5 Jahre
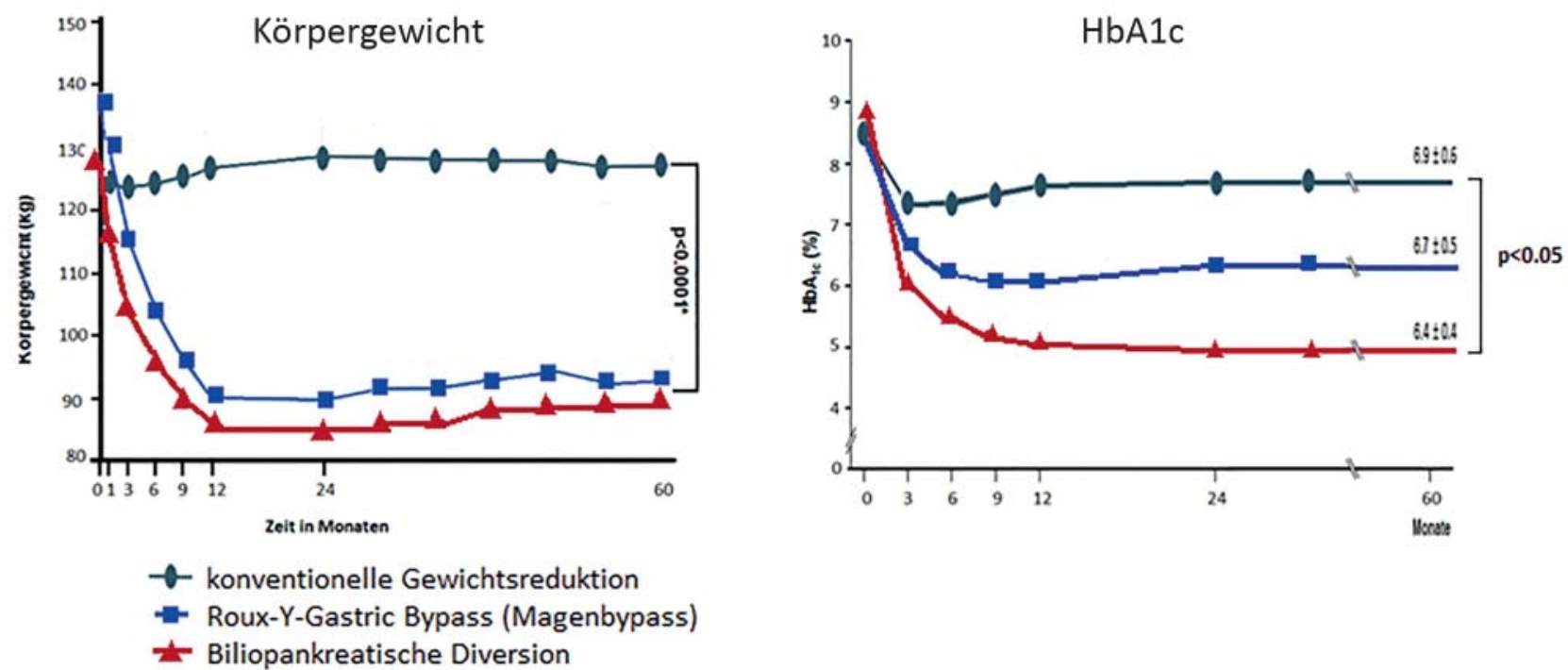

- Abb. 2 Anthropometrische Daten sowie Verlauf von Körpergewicht und HbA1c über 5 Jahre der Patienten der Studie von Mingrone et al. (nach $[9,10])$.

\section{Diabetes-Surgery-Studie (DSS)}

Die Autoren dieser Studie [12] haben einen etwas anderen Ansatz gewählt als die bisher zitierten Studien. In eine randomisierte kontrollierte Studie mit Zentren in den USA und Taiwan wurden zwischen 2008 und 2011 schließlich 120 Patienten mit einem BMI zwischen 30,0 und $39,9 \mathrm{~kg} / \mathrm{m}^{2}$ und einem HbA1c $\geq 8 \%$ einbezogen. Alle Patienten erhielten eine intensive Lebensstil- und medikamentöse Behandlung (LS/IMM). 60 Patienten wurden zusätzlich einer bariatrischen Operation unterzogen (RYGB). Die Lebensstil-/ medikamentöse Intervention wurde über 24 Monate intensiv durchgeführt, danach wurden die Patienten für ein weiteres Jahr von ihrem Hausarzt behandelt. Die Analyse der Daten erfolgte nach 36 Monaten. Als Endpunkt wurde ein Composite aus HbA1c $<7 \%$, LDL-Cholesterol $<2,6 \mathrm{mmol} / \mathrm{l}$ und systolischem Blutdruck $<130 \mathrm{mmHg}$ gewählt. Nach 3 Jahren wurde dieser primäre Endpunkt von $9 \%$ der LS/IMM- und $28 \%$ der LS/IMM+RYGB-Gruppe erreicht. Der mittlere HbA1c war 8,6 vs. 6,7\%. Eine Diabetesremission war bei keinem Patienten der LS/IMM-Gruppe zu verzeichnen, in der Gruppe mit zusätzlichem Magenbypass bei $17 \%$. Die Patienten hatten eine erstaunlich lange Diabetesdauer (9,1 vs. 8,9 Jahre) und trotzdem relativ hohe Nüchtern-C-Peptid-Spiegel (3,0 vs. $2,7 \mathrm{mg} / \mathrm{l})$. Trotz dieser ungünstigen Ausgangsbedingungen hatten in der Gruppe der LS/IMM + RYGB 73\% der Patienten nach einem Jahr einen $\mathrm{Hba}_{1 \mathrm{c}}<7 \%$ und nach 3 Jahren immer noch $58 \%$. Die Autoren konnten keine Korrelation zwischen Diabetesdauer, C-Peptid, HbA1c oder BMI und dem Erreichen des Composite-Endpunkts finden. Dies scheint der klinischen Erfahrung zumindest unserer Arbeitsgruppe und vieler Literaturdaten zu widersprechen.

Trotz aller Limitationen belegt die DSS den Nutzen der bariatrischen Chirurgie auch bei Typ-2-Diabetikern mit längerer Diabetesdauer. 
A Basisdaten

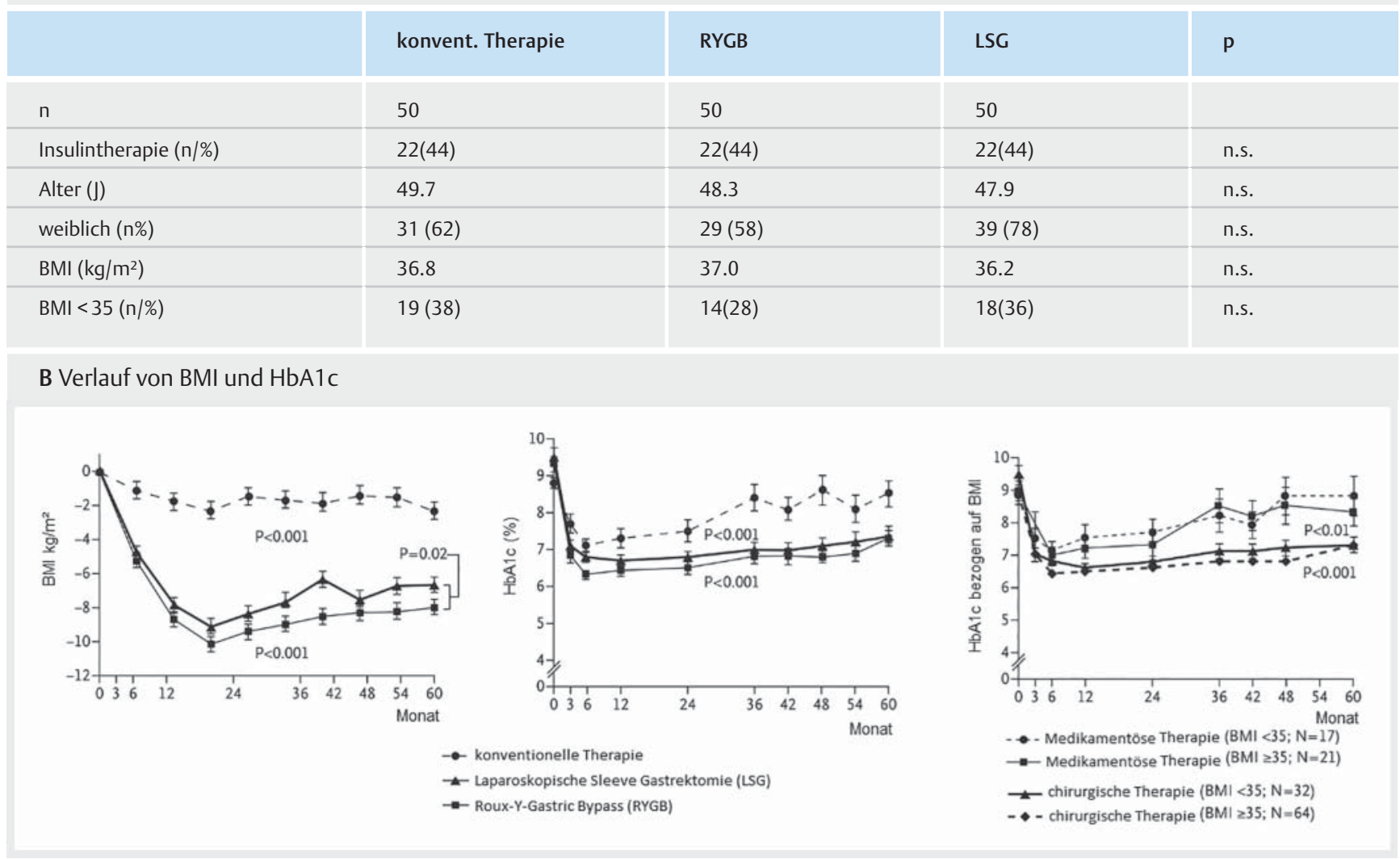

- Abb. 3 Basisdaten sowie Verlauf von BMI und HbA1c über 5 Jahre der Patienten der STAMPEDE-Studie von Schauer et al. (nach [7, 8]).

\section{Mikrovaskuläre Komplikationen bei Patien- ten mit Typ-2-Diabetes nach bariatrischer Chirurgie reduziert? Gibt es einen Legacy- Effekt der chirurgischen Gewichtsreduk- tion?}

Coleman et al. [19] gingen in einer großen retrospektiven Beobachtungsstudie anhand von Versicherungsdaten der Frage nach, ob eine Diabetesremission nach bariatrischer Chirurgie das Risiko inzidenter mikrovaskulärer Komplikationen reduziert. Die Autoren verfolgten 4683 Patienten im Alter vom 20 - 79 Jahren nach bariatrischer Chirurgie (79\% RYGB, 15\% LSG, 6\% AGB). Als primärer Endpunkt wurde ein Composite-Indikator aus dem ersten Auftreten von Retinopathie, Neuropathie und/oder Nephropathie nach Diabetesremission gewählt. Patienten mit Diabetesremission hatten ein um 29 \% geringeres Risiko für das erste Auftreten einer mikrovaskulären Komplikation gegenüber den Probanden, die keine Remission erreichten. Für die Gruppe der Diabetiker, die nach Remission ein Rezidiv erfuhren, war die Zeitdauer der Remission invers bezogen zur Inzidenz von Retinopathie, Neuropathie und/ oder Nephropathie. Jedes Jahr, das diese Patienten in der Diabetesremission verbrachten, war mit einer Reduktion des Risikos mikrovaskulärer Komplikationen um 19\% verbunden. Die Autoren schlussfolgern, dass es sich um einen „Legacy“-Effekt der bariatri-

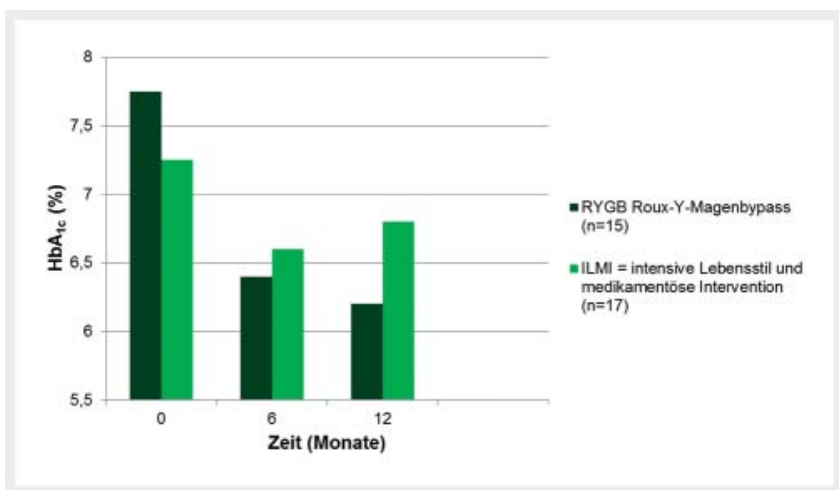

- Abb. 4 HbA1c im Verlauf von 12 Monaten der CROSSROADSStudie (nach [11]).

schen Chirurgie handelt in Anlehnung an den Begriff aus der DCCT-EDIC Studie [20].

Hauptsächlich wurde das Retinopathierisiko reduziert. Die Studie weist eine Vielzahl von Limitationen auf, an der Grundaussage dürfte aber kaum Zweifel bestehen, insbesondere da eine Vielzahl kleinerer Studien ebenso einen Effekt der bariatrischen Chirurgie auf die Reduktion des Risikos mikrovaskulärer Folgen und besonders der Retinopathie aufzeigen konnte [14, 21 - 23]. 


\section{Welche Faktoren beeinflussen die Remis- sion eines Diabetes mellitus Typ 2 nach bariatrischer Chirurgie?}

Die Verbesserung der Glykämie nach bariatrischer Chirurgie ist umso ausgeprägter, wenn

- die Erkrankung < 5 Jahre besteht,

- der Patient unter 40 - 50 Jahre alt ist,

- noch kein Insulin verwendet wurde und

- die Gewichtsreduktion sehr ausgeprägt ist [24-26].

Von Lee et al. [27] wurde ein Diabetes Surgery Score vorgeschlagen, der mit einem Score $>8$ eine Diabetesremission sehr wahrscheinlich macht bzw. mit einem Score $<4$ diese eher als wenig wahrscheinlich vorhersagen lässt ( $\bullet$ Tab. 2).

Ein weiterer prädiktiver Score für die Vorhersage der Wahrscheinlichkeit einer Diabetesremission nach RYGB wurde von Still et al. [28] als DiaRem Score publiziert. In diesen Score gehen vor allem das Alter und der präoperative HbA1c-Wert ein, darüber hinaus die Medikation mit oralen Antidiabetika und Insulin. Der DiaRem Score hat einen Bereich von 0-22 Punkten und wird in 5 Gruppen unterteilt:

0 - 2: höchste Wahrscheinlichkeit einer Remission mit 88 $99 \%, 3-7$ (64-88\%), $8-12$ (23-49\%), $13-17$ (11-33\%) und $18-22(2-16 \%)$, niedrigste Wahrscheinlichkeit einer Remission.

\section{Mechanismen der Verbesserung der diabe- tischen Stoffwechsellage und potenzielle Mechanismen einer Diabetesremission}

Von Malin et al. [25] werden die Mechanismen, die einer Diabetesremission nach bariatrischer Chirurgie zugrunde liegen können, ausführlich beschrieben. - Tab. 3 bietet eine Zusammenfassung dieser Mechanismen.

W. Pories betonte bereits 1992 [2], dass die Verbesserung der diabetischen Stoffwechsellage in erster Linie durch die Gewichtsreduktion zu erklären ist. Dies gilt auch heute noch. Vor über 20 Jahren hatte diese Arbeitsgruppe aber bereits erkannt, dass die Modifikation gastrointestinaler Faktoren an der metabolischen Verbesserung beteiligt sein muss. Die Rolle des Gastrointestinaltrakts im Rahmen der Regulation der Energie- und Glukosehomöostase ist inzwischen zu einem Hauptfeld der „bariatrischen“ Forschung geworden. Diese Dinge werden im Folgenden kurz angerissen.

\section{Kalorische Restriktion (und) Gewichtsverlust}

Die Besserung der Glukosehomöostase nach bariatrischen Operationen (RYGB, LSG, BPD) tritt typischerweise innerhalb weniger Tage nach OP auf, ehe ein signifikanter Gewichtsverlust eintritt. Somit kann man schlussfolgern, dass der Gewichtsverlust per se wahrscheinlich nicht die wesentliche Rolle als Mechanismus einer verbesserten Glykämie p. o. haben dürfte. Außerdem spielt offenbar die Art der bariatrischen Operation eine Rolle hinsichtlich der Geschwindigkeit der Verbesserung der Glukosetoleranz. Beispiels- weise ist das Ausmaß der Kalorienbeschränkung nach Magenbypass offenbar größer als nach Magenband (AGB). Darüber hinaus zieht der Magenbypass eine rasche Passage oraler Nährstoffe in dem Darm nach sich, mit der Folge von frühzeitig hohen Peaks an Glukose, GLP-1 und Insulin [29]. Vergleicht man Patienten mit Magenbypass mit Patienten unter einer VLCD (very low calorie diet), zeigt sich, dass beide Verfahren vergleichbare Verbesserungen der Insulinsekretion und -sensitivität bewirken [30]. Da der direkte Vergleich von VLCD und RYGB eher problematisch erscheint, wurden von Lingvay et al. [31] VLCD und Magenbypass bei Diabetikern verglichen, die als ihre eigenen Kontrollen dienten. Nach 10 Tagen VLCD zeigt sich eine signifikante Verbesserung von NBZ, Peak-Glukose und der Glukosefläche unter der Kurve, nicht jedoch unter RYGB, und dies trotz größerer GLP-1Antwort unter RYGB.

Studien zur Wirkung sehr niedrigkalorischer Diäten (VLCD) bei Diabetes mellitus Typ 2 sind sehr viele durchgeführt worden. Steven et al. [32] gehen nun in einem interessanten Ansatz erneut dieser Frage nach: 30 Patienten mit Typ-2-Diabetes wurden in einer dreistufigen Studie untersucht.

1. 8 Wochen VLCD mit ca. $700 \mathrm{kcal} /$ die in Form von Optifast ${ }^{\circledR}$

2. schrittweiser Übergang zu einer isokalorischen Kost über 14 Tage

3. strukturiertes, individualisiertes Programm zur Erhaltung des reduzierten Gewichts

Die isokalorische Nahrungszufuhr wurde anhand der Grundumsatzmessung mit der indirekten Kalorimetrie ermittelt. Die Autoren klassifizierten Patienten, die nach Rückkehr zur isokalorischen Kost einen Blutzucker $<7$ mmol/l aufwiesen, als „Responder“. Diese waren charakterisiert durch

a) Verbesserung der Insulinsekretion, z. T. mit Rückkehr der 1. Phase der Insulinsekretion,

b) Reduktion der Insulinresistenz,

c) Normalisierung des hepatischen Fettanteils mit einem $20 \%$ igen Abfall der Synthese von VLDL1-TG,

d) Reduktion des pankreatischen Fettdepots (annehmbar als Folge von c).

Dieses Programm war über 6 Monate erfolgreich. Es bleibt die Frage, ob eine echte Langzeitremission des Typ-2-Diabetes so möglich ist. Die Charakteristika der „Responder“ entsprechen den metabolischen Ergebnissen der bariatrischen Chirurgie bei Diabetes mellitus Typ 2, dort aber mit Verläufen von bis zu 5 Jahren.

Von Khoo et al. [33] wird eine hinsichtlich der Fallzahl sehr übersichtliche Studie zum direkten Vergleich von RYGB $(n=10)$ und kalorischer Restriktion $(n=10)$ vorgelegt. Es handelt sich um die erste Studie, die über ein breites Spektrum metabolischer Veränderungen früh nach RYGB im Vergleich zu kalorischer Restriktion berichtet. Die Autoren stellen fest, dass sich nach RYGB eine verbesserte „metabolische Flexibilität“ einstellt, ersichtlich an einem gesteigerten Disposal von Glukose und Aminosäuren sowie einer kompletteren ß-Oxidation der Fettsäuren im Vergleich zur kalorischen Restriktion.

Aus diesen und weiteren Untersuchungen ergeben sich die Hinweise darauf, dass die kalorische Restriktion per se verantwortlich zu sein scheint für die rasche Verbesserung der Glykämie nach bariatrischer Operation und dass durch eine allerdings lang- 
- Tab. 2 Diabetes Surgery Score (modifiziert nach Lee et al. [27]).

\begin{tabular}{|l|l|l|l|l|}
\hline & \multicolumn{2}{|l|}{ Diabetes Surgery Score } & & $\mathbf{3}$ \\
\hline & $\mathbf{0}$ & $\mathbf{1}$ & $\mathbf{2}$ & $>50$ \\
\hline BMl $\left(\mathrm{kg} / \mathrm{m}^{2}\right)$ & $<30$ & $30-39$ & $40-49$ & $>6,0$ \\
\hline C-Peptid $(\mathrm{ng} / \mathrm{ml})$ & $0,9-1,9$ & $2,0-3,9$ & $4,0-6,0$ & $<2$ \\
\hline Diabetesdauer $(\mathrm{J})$ & $>10$ & $5-10$ & $2-4,9$ & \\
\hline Alter $(<40$ vs. $>40)$ & $\geq 40$ & $<40$ & & \\
\hline
\end{tabular}

Diabetesremission wahrscheinlich: DSS $>8$. Diabetesremission wenig wahrscheinlich: DSS $<4$.

- Tab. 3 Mechanismen der Diabetesremission im Gefolge einer bariatrisch-chirurgischen Therapie.

\section{A Zusammenfassung der antidiabetischen Effekte verschiedener bariatrischer Eingriffe}

\begin{tabular}{|c|c|c|c|}
\hline & LSG & RYGB & BPD \\
\hline Tempo der Diabetesremission & rasch & rasch & rasch \\
\hline Insulinsensitivität & verbessert & verbessert & supranormal \\
\hline Insulinsekretion & verbessert & verbessert & reduziert \\
\hline Ghrelin & $\downarrow$ & $\uparrow \downarrow$ & $\downarrow$ \\
\hline GLP 1 & $\uparrow$ & $\uparrow$ & $\uparrow$ \\
\hline PYY & $\uparrow$ & $\uparrow \uparrow \uparrow$ & $\uparrow$ \\
\hline \multicolumn{4}{|c|}{ B Potenzielle Mechanismen der Diabetesremission (nach [25]) } \\
\hline \multicolumn{4}{|l|}{ Klinische Charakteristika } \\
\hline \multicolumn{4}{|l|}{ junges Alter } \\
\hline \multicolumn{4}{|l|}{ kürzere Diabetesdauer } \\
\hline \multicolumn{4}{|l|}{ wenige Antidiabetika } \\
\hline \multicolumn{4}{|l|}{ hoher Gewichtsverlust } \\
\hline \multicolumn{4}{|l|}{ Metabolische Charakteristika } \\
\hline \multicolumn{4}{|l|}{ C-Peptid $\uparrow$} \\
\hline \multicolumn{4}{|l|}{ Adiponektin $\uparrow \uparrow$} \\
\hline \multicolumn{4}{|l|}{$\mathrm{CRP} \downarrow \downarrow$} \\
\hline \multicolumn{4}{|l|}{ TNF $\alpha$ und Leptin $\downarrow \leftrightarrow$} \\
\hline \multicolumn{4}{|l|}{ GLP-1 $\uparrow$} \\
\hline \multicolumn{4}{|l|}{$\mathrm{GIP} \leftrightarrow$} \\
\hline \multicolumn{4}{|l|}{ Ghrelin $\downarrow$} \\
\hline ß-Zell-Funktion $\uparrow \uparrow$ & & & \\
\hline
\end{tabular}

LSG: Laparoskopische Sleeve-Gastrektomie („Schlauchmagen“); RYGB: Roux-Y-Gastric Bypass (Magenbypass); BPD: Biliopankreatische Diversion.

fristig schwer umsetzbare kalorische Restriktion ähnliche Verbesserungen der Glykämie wie unter bariatrischen Operationen zu erzielen wären [34, 35]

\section{Gewichtsreduktion verbessert Insulinsensitivität und Insulinsekretion}

Der Hauptteil der Effekte, die zu einer Remission des Typ-2-Diabetes führen, scheint innerhalb von 8 Wochen postoperativ abzulaufen. 
Es kommt zu einer deutlichen Verbesserung der Insulinsensitivität und der Insulinsekretionsrate. In einem ausgefeilten Design konnte die Gruppe um G. Mingrone [36] mittels euglykämischhyperinsulinämischer Clamp-Technik, ivGTT und oGTT zeigen, dass 1 Monat nach RYGB

- die Insulinsekretionsrate im Vergleich zum Status vor der Operation deutlich angestiegen war,

- bei Patienten mit Typ-2-Diabetes die erste Phase der Insulinsekretion sich wiederherstellen ließ,

- die Insulinsensitivität im Clamp deutlich verbessert wurde und

- GLP-1 sowie GIP deutlich anstiegen, und dies vor allem bei Patienten mit Typ-2-Diabetes.

Einige Studien zeigen aber auch, dass die Verbesserung der Insulinsensitivität längere Zeiträume benötigt und zumindest teilweise zur Reduktion des Körpergewichts in Beziehung steht [34]. Beispielsweise könnte GLP-1 langfristig die Regeneration von ß-Zellen stimulieren oder gar zu deren Hypertrophie führen [34]. Allerdings wird die Rolle des Gewichtsverlusts bei der initialen Remission und dem langfristigen Erhalt der Remission sehr kontrovers diskutiert. Dauer und Schweregrad des Diabetes sowie individuelle Patientencharakteristika dürften diese Prozesse beeinflussen. Es soll hier besonders hervorgehoben werden, dass eine Gewichtsreduktion mittels VLCD (,konventionell“) nicht mit der unter bariatrischen Operationen gleichzusetzen ist. Der durch eine VLCD induzierte Gewichtsverlust führt immer zu kompensatorischen Veränderungen der Homöostase, die verbunden sind mit einem Anstieg des Hungergefühls, deutlich gesteigertem Ghrelin und reduzierten GLP-1 und PYY. Diese Mechanismen sind verantwortlich für die extrem hohe Rate von Rezidiven nach „konventioneller" Gewichtsreduktion [35], von vielen Autoren als Jo-jo-Effekt bezeichnet. Im Gegensatz dazu steigen nach bariatrischen Operationen GLP-1- und PYY-Spiegel an, während Ghrelin meist abfällt. Diese Veränderungen sind offenbar für das reduzierte Appetitverhalten, das anhaltenden Gewichtsverlust und Gewichtserhalt fördert, verantwortlich. Es wäre wünschenswert, wenn diese biochemischen Mechanismen von den Verantwortlichen (Entscheidungsträgern) bei Krankenkassen und Politik verstanden werden würden.

Die Gewichtsreduktion nach RYGB führt auch zu einer Optimierung der Insulin-Signaltransduktion an Muskulatur und Fettgewebe. Albers et al. [37] entnahmen Muskel- und Fettgewebsbiopsien während euglykämisch-hyperinsulinämischer Clamps bei normoglykämischen Adipösen und Patienten mit Typ2-Diabetes vor RYGB, 1 Woche, 3 Monate bzw. 12 Monate postoperativ. Die Autoren konnten die größte Verbesserung der Insulinsensitivität aufzeigen, wenn der Gewichtsverlust maximal war. Parallel dazu stieg Adiponectin signifikant an und an der Muskulatur ließ sich eine deutliche Steigerung der Proteinexpression des Glukosetransporters GLUT 4 nachweisen. Die Autoren schlussfolgern, dass sich nach RYGB eine Veränderung des Phänotyps der Muskulatur und des Fettgewebes einstellt, der sich vor allem durch höhere Insulinsensitivität auszeichnet.

Von Dadson et al. [38] wird in einem sehr ausgefeilten Studiendesign unter Nutzung der Bestimmung der Körperfettverteilung mittels MRI und markierter Glukose (PET) der gewebespezifische Glukose-Uptake vor und nach bariatrischen Verfahren (RYGB und
LSG) ermittelt. Auch diese Daten tragen zum besseren Verständnis der molekularen Wirkungen der bariatrischen Chirurgie bei.

\section{Reduktion der hepatischen und pankreatischen Fettdepots, Reduktion der freien Fettsäuren.}

Patienten mit Typ-2-Diabetes haben vermehrte hepatische und pankreatische Fettdepots verglichen mit normoglykämischen Probanden mit vergleichbarem BMI [39].

Innerhalb von 1 Woche nach Magenbypass oder VLCD fällt der hepatische Lipidgehalt rasch und steht in enger Verbindung zur verbesserten hepatischen Insulinsensitivität [40]. Der Abbau der pankreatischen Fettdepots hin zu nichtdiabetischen Levels braucht mit ca. 8 Wochen deutlich mehr Zeit, ist aber mit einer Wiederherstellung der 1 . Phase der Insulinsekretion verbunden [34, 36, 40].

In der Pathogenese der Insulinresistenz und des Typ-2-Diabetes spielen freie Fettsäuren eine wesentliche Rolle. Nach bariatrischen Operationen fallen FFS deutlich ab [41, 42], insbesondere nach RYGB [43]. Von Hoffstedt et al. [41] wird nun auch der Metabolismus des Fettgewebes vor und bis zu 5 Jahre nach RYGB bei adipösen Frauen untersucht. Dabei zeigten sich neben einer signifikanten Verbesserung der Insulinsensitivität (HOMA) eine Reduktion der Fettzellgröße (bei unveränderter Fettzellzahl) und eine reduzierte Fettgewebslipolyse mit konsekutiver Reduktion der FFS.

\section{Reduktion der „Inflammation des Fettgewebes“?}

Bisher galt als relativ gesichert, dass es im Gefolge einer deutlichen Gewichtsreduktion, u. a. nach einer bariatrischen Operation, zu einer Reduktion der Inflammation des Fettgewebes kommen würde. Dabei wurden sehr verschiedene Marker der „Inflammation" gemessen, allerdings bei in der Regel sehr kleinen Fallzahlen. Dabei zeigte sich ein Abfall für IL-6 und TNF $\alpha$ [44-46].

Aus der Gruppe von D. Cummings wird nun eine Teilauswertung der CROSSROADS-Studie vorgelegt [47]. Dabei wurden 16 adipöse Patienten mit Typ-2-Diabetes randomisiert einem RYGB oder der intensiven Lebensstil- und medizinischen Therapie (ILMI) unterzogen. Die Auswertung erfolgte in beiden Gruppen nach einem Gewichtsverlust von $7 \%$. Die Inflammation des Fettgewebes wurde anhand von Ganzkörper-Genexpressionsanalysen und der Flowzytometrie der Gewebsleukozyten gemessen. Nach $7 \%$ Gewichtsverlust war unter RYGB eine Zunahme der Fettgewebsinflammation zu verzeichnen, unter ILMI wurden keine wesentlichen Veränderungen beobachtet. Die Autoren schlussfolgern, dass eine Reduktion der Fettgewebsinflammation kein Mechanismus ist, durch den der RYGB eine gewichtsunabhängige antidiabetische Wirkung entfalten kann.

\section{Veränderungen der enteroinsulinären Achsen}

Langzeitdaten zu den Effekten der bariatrischen Chirurgie auf Inkretine und mehr noch Adipokine sind derzeit noch rar. Kurzfristige Effekte sind dagegen gut untersucht, wenn auch meist an sehr kleinen Fallzahlen. Die laparoskopische Schlauchmagenbildung ist inzwischen zu einer der am häufigsten ausgeführten bariatrischen Operationen in vielen Ländern geworden, wobei noch wenige Daten zu den biochemischen Markern vorliegen. 
GLP-1

Nach bariatrischen Operationen (RYGB, LSG, BPD) sind die GLP-1Spiegel postprandial deutlich erhöht. Parallel dazu finden sich deutlich erhöhte postprandiale Insulinspitzen [48 - 50]. Die überschießende postprandiale GLP-1-Sekretion ist ab dem 2. postoperativen Tag nachweisbar und persistiert langfristig.

Unter diätetischer kalorischer Restriktion bleiben die GLP-1Spiegel dagegen unverändert und dies trotz z. T. vergleichbarer Gewichtsreduktion. Die überschießende GLP-1-Sekretion nach bariatrischen Operationen scheint zumindest z. T. für die hyperinsulinämischen Hypoglykämien verantwortlich zu sein, die bei einem kleinen Teil dieser Patienten auftreten können [51]. Der GLP-1-Antagonist Exendin [9-39] eliminiert die abnorm hohe Insulinsekretion dieser Patienten und verhindert die sog. postbariatrischen Hypoglykämien [52].

Gegenstand der Debatte ist auch, ob GLP-1 bzw. die bariatrische Prozedur des Magenbypass die $\beta$-Zellmasse beeinflussen kann. Es gibt Autoren, die über eine Zunahme der $\beta$-Zellmasse berichten, und Berichte, die dies nicht bestätigen [53 - 57]. Der GLP-1-Anstieg soll nach RYGB deutlicher ausfallen als nach LSG [58]. Madsbad und Holst [59] heben hervor, dass GLP-1 natürlich ein wichtiger Mediator der Remission eines Typ-2-Diabetes nach RYGB oder LSG ist, dass eine ausreichende funktionelle sekretorische Kapazität der $\beta$-Zellen (d. h. eine ausreichende Restsekretion von Insulin) eine Voraussetzung für die Remission ist. Beachtet werden sollte auch, dass die Sekretion von GIP nach RYGB nahezu unbeeinflusst bleibt, nach LSG aber deutlich ansteigt und nicht durch Exendin [9-39] antagonisiert wird [60]. Zusammenfassend spielt GLP-1 im Orchester der postbariatrischen Veränderungen der enteroinsulinären Achse eine entscheidende Rolle. Trotzdem kann auch eine solch ausgeprägte antidiabetische Wirkung ein $\beta$-Zellversagen nicht reversibel gestalten [59].

\section{Peptid YY (PYY) „Sättigungshormon“}

PYY wird co-sezerniert mit GLP-1 in den L-Zellen des distalen Jejunums auf Nahrungsreiz. Nach bariatrischen Operationen (RYGB, LSG) steigen GLP-1 und PYY deutlich an, wobei der PYY-Anstieg nach RYGB wesentlich ausgeprägter sein soll. Diese hormonellen Veränderungen tragen zum reduzierten Appetit bei und fördern den langfristigen Gewichtsabbau bzw. die Aufrechterhaltung des reduzierten Gewichts [58, $61-65]$.

\section{Ghrelin}

Ghrelin (unser „Hungerhormon“) wird im Magenfundus, proximalen Dünndarm und Pankreas sezerniert. Über hypothalamische Rezeptoren wird der Appetit stimuliert. Ghrelin hemmt die Insulinsekretion, supprimiert Adiponektin. Nach bariatrischen Operationen fällt Ghrelin deutlich ab. Die Arbeiten von Cummings et al. über $24 \mathrm{~h}$ Ghrelin-Plasmaspiegel nach RYGB zeigen dies sehr eindrucksvoll [66] ( $\mathbf{A b b . 5 )}$. Von Ochner et al. wurde der Abfall des Ghrelin-Spiegels gar als Marker für den Erfolg der bariatrischen Chirurgie gekennzeichnet [67]. In einer rezenten indischen Studie wurde gezeigt, dass nach LSG die AUC (area under the curve) für Ghrelin nach 6 Monaten um $73 \%$ und für Leptin um $50 \%$ abfiel [68]. Auch wenn fast alle diese Studien [42, 68 - 70] mit sehr kleinen Fallzahlen operieren, kann inzwischen als gesichert gelten, dass Ghrelin unter LSG deutlich mehr abfällt als unter RYGB. - Tab. 3 zeigt zusammenfassend die antidiabetischen Effekte verschiedener bariatrischer Eingriffe.

Interessante neue Daten, die mittels funktioneller MRI erhoben wurden, zeigen, dass Adipöse nach RYGB im Vergleich zu Patienten nach Magenband sehr differente Reaktionen verschiedener Hirnregionen aufweisen, die letztlich für Hunger und Sättigung verantwortlich sind [71]. Es zeigte sich aber auch ein Shift der Präferenzen für Nahrung hin zu weniger kalorienreichen und weniger fetthaltigen Nahrungsmitteln, und dieser Shift war unabhängig von Hunger bzw. Sättigung. Die Veränderungen im Metabolismus der Gallensäuren könnten in diesem Zusammenhang eine Rolle spielen.

\section{Fettgewebshormone/Adipokine}

Daten zu den Adipokinen beziehen sich fast ausschließlich auf Adiponektin und Leptin. In der Regel wird bereits kurz nach RYGB ein Anstieg von Adiponektin beobachtet [25, 41, 44, 72]. Malin et al. [25] schlussfolgern, dass ein geringer Anstieg von Adiponektin nach RYGB oder LSG bei Patienten mit Typ-2-Diabetes die Nichtremission vorhersagen könnte. Bisher werden nur in der Studie von Hoffstedt et al. [41] 5-Jahres-Daten zu Adiponektin nach RYGB vorgelegt. Dabei zeigte sich, dass der nach 2 Jahren zu verzeichnende Adiponektinanstieg auch nach 5 Jahren noch in gleichem Ausmaß vorhanden war.

Der Leptinspiegel fällt nach bariatrischen Operationen in der Regel ab [25, 42, 44-46]. Ob der Abfall des Leptinspiegels allein auf den Verlust an Fettmasse nach bariatrischer Operation zurückzuführen ist, oder ob tatsächlich eine Zunahme der Leptinsensitivität eintritt, ist noch nicht endgültig geklärt [73].

\section{Rolle von Duodenum und proximalem Jejunum (Foregut-Hypothese)}

Magenbypass und biliopankreatische Diversion bewirken u. a. die Ausschaltung von Duodenum und proximalem Jejunum aus dem Verdauungsprozess. Parallel dazu werden inkomplett verdaute Nahrungsbestandteile sehr rasch in tiefere Darmabschnitte transportiert. Die Foregut-Hypothese postuliert, dass der Ausschluss von Duodenum und proximalem Jejunum aus dem Verdauungsprozess den Anstieg eines Anti-Inkretinsignals vermindert bzw. eliminiert, das normalerweise als Gegenspieler der inkretinvermittelten Insulinsekretion wirkt und postprandiale Hypoglykämien verhindern könnte. Insbesondere die experimentelle Methode des duodenojejunalen Bypass (DJB) wurde von verschiedenen Arbeitsgruppen genutzt, um die postulierten Mechanismen zu verifizieren [74-77]. Von einem ähnlichen Konzept geht auch die Anwendung des „Endobarriers“ aus.

All diese Studien zeigen, dass ein Bypass eines Segments des proximalen Dünndarms (Duodenum und proximales Jejunum) die Glukosetoleranz rasant bessert und dies unabhängig von Nahrungszufuhr, Körpergewicht oder Malabsorption.

Die Umgehung von Duodenum und proximalem Jejunum führt ZU: 


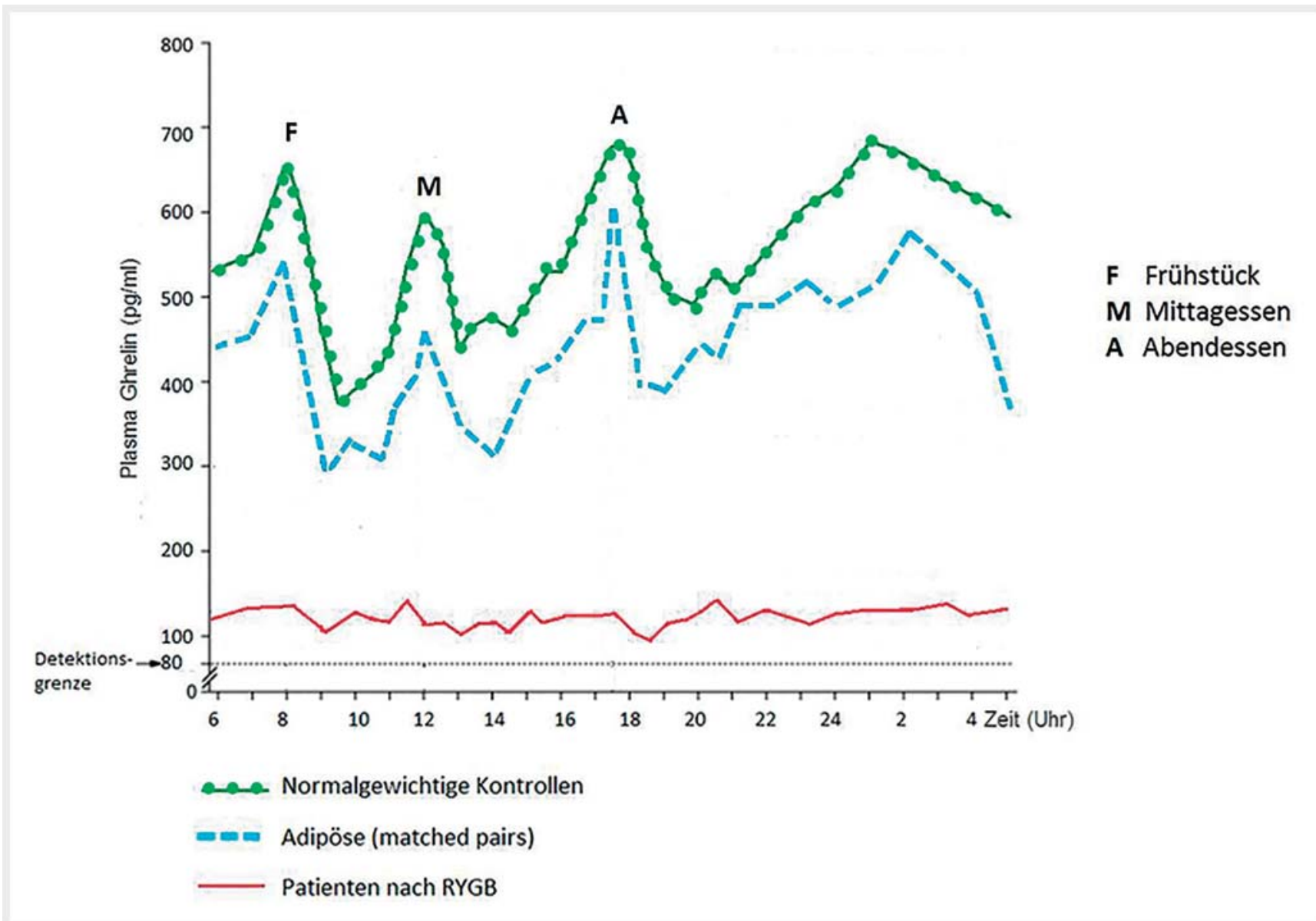

- Abb. 5 Ghrelin-Plasmaspiegel bei Normalgewichtigen, Adipösen und Patienten nach RYGB (nach [66]).

- Besserung der Insulinsensitivität (je länger der jejunale Bypass, desto mehr bessert sich die Insulinsensitivität)

- Hemmung der Lipolyse

- Förderung der Insulin-Clearance

\section{Intestinale Adaptation}

Bariatrische Operationen führen zu ausgeprägten intestinalen Adaptationen an die veränderte Anatomie [29]. Diese intestinale Adaptation scheint zur verbesserten Glukosehomöostase beizutragen. Was ist gemeint?

Nach einer laparoskopischen Magenbypass-Operation macht der alimentäre Schenkel eine Hypertrophie und Hyperplasie des Epithels durch, verbunden mit einer vermehrten Expression von Glukosetransportern und einem gesteigerten Glukose-Uptake in die intestinalen Epithelzellen. Auch die Zahl der Zellen, die GLP-1 und GIP im alimentären Schenkel produzieren, nimmt zu [60, 78].

Im Gegensatz dazu wurde nach Sleeve-Gastrektomie keine Hyperplasie des nachgeschalteten Intestinums gefunden. Bei Nagetieren wurde allerdings auch eine Zunahme der Zahl und Dichte GLP-1 enthaltender Zellen nach LSG gefunden. Im Gefolge einer LSG ist darüber hinaus die intestinale Glukoseabsorption reduziert [78]. Nach den genannten bariatrischen Operationen wird die Glukosehomöostase demzufolge durch unterschiedliche Mechanismen, die sich z. T. überlappen, verbessert [60].

\section{Gallensäuren, FGF-19 und das Darm-Mikrobiom}

Einer der aktuellen Hotspots der bariatrischen Forschung stellt die Verknüpfung von Gallensäuren (GS), FGF-19 und dem DarmMikrobiom dar.

Nach RYGB und LSG steigen zirkulierende GS an, und dies ist assoziiert mit verbesserter Glukosetoleranz. Der Fibroblast Growth Factor 19 (FGF-19) wird im Ileum produziert, stimuliert durch die GS-Absorption, wirkt als Hormon und reguliert auch die Synthese der GS mit weiteren Effekten auf den Glukose- und Lipidstoffwechsel [79].

Auch FGF-19 steigt nach RYGB und LSG an [80], nicht jedoch nach diätetischer kalorischer Restriktion oder Magenband [81]. Eine mögliche Erklärung wäre, dass die anatomischen Veränderungen nach RYGB zu einer verzögerten Vermischung von GS und der aufgenommenen Nahrung führen, was letztlich den Anstieg der zirkulierenden GS und FGF-19 verursachen könnte [29].

Im Gegensatz zu Nichtdiabetikern weisen Typ-2-Diabetiker unabhängig vom BMI verminderte Spiegel an GS und FGF-19 auf. Typ-2-Diabetiker, die nach RYGB eine Diabetesremission erreichen 
konnten, zeigten höhere GS- und FGF-19-Spiegel als Diabetiker, die keine Remission erreichten [82]. Eine Vielzahl von Untersuchungen geht dem Link zwischen Typ-2-Diabetes bzw. Insulinresistenz, GS und FGF-19 nach.

Seit einiger Zeit wird diskutiert, dass bestimmte Veränderungen der Zusammensetzung der Mikroorganismen des Darms („Mikrobiota“: Bakterien, Archaeen, Viren) adipogen sein sollen. Klare Daten dazu stehen aus. Die Gesamtheit der Gene, die die Mikrobiota kodieren und die der Darm enthält, wird als DarmMikrobiom bezeichnet.

Bariatrische Operationen, diätetische kalorische Restriktionen und die Veränderungen des GS-Metabolismus können die DarmMikrobiota von einem „adipösen“ Profil (mit hohen Quotienten von Firmicutes/Bacteriodetes) zu einem „schlankeren“ Profil modulieren. Bacteriodetes spielen eine Hauptrolle im Prozess der Dekonjungation von GS und verändern offenbar auch die GSZusammensetzung. In der Frühphase nach RYGB findet sich ein Peak von sekundären GS wie Ursodeoxycholsäure, später steigen die primären GS an [83]. Diese Veränderungen des GS-Metabolismus können die beschriebenen frühen Verbesserungen der Insulinsensitivität nach RYGB mitbegründen.

Bariatrische Operationen bewirken demzufolge einen Anstieg von GLP-1, GS und FGF-19, beeinflussen die intestinale Glukoseverwertung und die Darm-Mikrobiota. Das komplexe Zusammenspiel dieser Mechanismen scheint für die antidiabetischen Effekte von RYGB und LSG mit verantwortlich zu sein.

\section{Schlussfolgerungen}

Ist Diabetes heilbar durch bariatrische Chirurgie? Die klare Antwort muss „Nein“ lauten, aber:

1. 35 - $50 \%$ der Patienten mit Typ-2-Diabetes, die mittels bariatrischer Chirurgie initial nach Operation eine Diabetesremission erreichen konnten, erleben im weiteren Verlauf das Wiederkehren der „Krankheit“, d. h. erneut ansteigende Blutzuckerwerte [84].

2. Die mittlere „krankheitsfreie Zeit“ beträgt 8,3 Jahre (Range 5 15 Jahre) [84].

3. Jedes Jahr, das die Patienten mit Typ-2-Diabetes in der Remission der Erkrankung verbrachten, war mit einer Reduktion des Risikos mikrovaskulärer Komplikationen um 19\% verbunden [19].

Insofern ist die bariatrische Chirurgie in Form von RYGB, LSG und den neueren Verfahren wie Omega-Loop-Bypass („Mini-Bypass“) oder der BPD eine wirkungsvolle therapeutische Option in der Behandlung des Diabetes mellitus Typ 2 und verringert offenbar auch das Risiko des Auftretens von mikrovaskulären Diabetesfolgekomplikationen. Je früher im Krankheitsverlauf die bariatrische Chirurgie herangezogen wird, desto effektiver scheinen diese Verfahren zu sein.

Welche der Operationen für Patienten mit Typ-2-Diabetes am besten geeignet ist, ist derzeit nicht definitiv entschieden. Der RYGB scheint etwas effektiver zu sein.
Interessenkonflikt

Die Autoren geben an, dass kein Interessenkonflikt besteht.

\section{Literatur}

[1] Wadden TA. Treatment of obesity by moderate and severe caloric restriction. Results of clinical research trials. Ann Intern Med 1993; 119: 688-693

[2] Pories W], MacDonald KG Jr, Flickinger EG et al. Is type II diabetes mellitus (NIDDM) a surgical disease? Ann Surg 1992; 215: 633-643

[3] Christou NV, Sampalis JS, Liberman M et al. Surgery decreases long-term mortality, morbidity, and health care use in morbidly obese patients. Ann Surg 2004; 240: 416-424

[4] Sjöström L. Review of the key results from the Swedish Obese Subjects (SOS) trial - a prospective controlled intervention study of bariatric surgery. J Intern Med 2013; 273: 219-234

[5] Romeo S, Maglio C, Burza MA et al. Cardiovascular events after bariatric surgery in obese subjects with type 2 diabetes. Diabetes Care 2012; 35: $2613-2617$

[6] Schauer PR, Kashyap SR, Wolski K et al. Bariatric surgery versus intensive medical therapy in obese patients with diabetes. N Engl J Med 2012; 366: $1567-1576$

[7] Schauer PR, Bhatt DL, Kirwan JP et al. Bariatric surgery versus intensive medical therapy for diabetes - 3-year outcomes. N Engl J Med 2014; 370: $2002-2013$

[8] Schauer PR, Bhatt DL, Kirwan JP et al. Bariatric surgery versus intensive medical therapy for diabetes - 5-year outcomes. N Engl J Med 2017; 376: $641-651$

[9] Mingrone G, Panunzi S, De Gaetano A et al. Bariatric surgery versus conventional medical therapy for type 2 diabetes. N Engl J Med 2012; 366 : $1577-1585$

[10] Mingrone G, Panunzi S, De Gaetano A et al. Bariatric-metabolic surgery versus conventional medical treatment in obese patients with type 2 diabetes: 5 year follow-up of an open-label, single-centre, randomised controlled trial. Lancet 2015; 386: 964-973

[11] Cummings DE, Arterburn DE, Westbrook EO et al. Gastric bypass surgery vs intensive lifestyle and medical intervention for type 2 diabetes: the CROSSROADS randomised controlled trial. Diabetologia 2016; 59: 945 953

[12] Ikramuddin S, Korner ], Lee W] et al. Durability of addition of Roux-en-Y gastric bypass to lifestyle intervention and medical management in achieving primary treatment goals for uncontrolled type 2 diabetes in mild to moderate obesity: a randomized control trial. Diabetes Care 2016; 39: $1510-1518$

[13] Buse JB, Caprio S, Cefalu WT et al. How do we define cure of diabetes? Diabetes Care 2009; 32: 2133-2135

[14] Sjöström L, Narbro K, Sjöström CD et al. Effects of bariatric surgery on mortality in Swedish Obese Subjects. N Engl J Med 2007; 357: 741 - 752

[15] Sjöholm K, Pajunen P, Jacobson P et al. Incidence and remission of type 2 diabetes in relation to degree of obesity at baseline and 2 year weight change: the Swedish Obese Subjects (SOS) study. Diabetologia 2015; 58: $1448-1553$

[16] Sjöström L, Peltonen M, Jacobson P et al. Association of bariatric surgery with long-term remission of type 2 diabetes and with microvascular and macrovascular complications. JAMA 2014; 311: 2297-2304

[17] Carlsson LMS, Peltonen M, Ahlin S et al. Bariatric surgery and prevention of type 2 diabetes in swedish obese subjects. N Engl J Med 2012; 367: $695-704$

[18] Sjöholm K, Sjöström E, Carlsson LMS et al. Weight change-adjusted effects of gastric bypass surgery on glucose metabolism: 2- and 10-year 
results from the Swedish Obese Subjects (SOS) Study. Diabetes Care 2016; 39: 625-631

[19] Coleman KJ, Haneuse S, Johnson E et al. Long-term microvascular disease outcomes in patients with type 2 diabetes after bariatric surgery: Evidence for the legacy effect of surgery. Diabetes Care 2016; 39: $1400-1407$

[20] Nathan DM, Engel S, Friday JB, The Diabetes Control and Complications Trial/Epidemiology of Diabetes Interventions and Complications (DCCT/ EDIC) Study Research Group et al. Intensive diabetes treatment and cardiovascular disease in patients with type 1 diabetes. N Engl J Med 2005; 353: $2643-2653$

[21] Banks ], Adams ST, Laughlan K et al. Roux-en-Y gastric bypass could slow progression of retinopathy in type 2 diabetes: a pilot study. Obes Surg 2015; 25: 777-781

[22] Brynskov T, Laugesen CS, Svenningsen AL et al. Monitoring of diabetic retinopathy in relation to bariatric surgery: a prospective observational study. Obes Surg 2016; 26: 1279-1286

[23] Amin AM, Wharton H, Clarke M et al. The impact of bariatric surgery on retinopathy in patients with type 2 diabetes: a retrospective cohort study. Surg Obes Relat Dis 2016; 12: 606-612

[24] Schulze zur Wiesch C, Lautenbach A, Aberle J. Adipositas - Neue Aspekte in der internistischen Betreuung vor und nach bariatrischer Chirurgie. Dtsch med Wochenschr 2016; 141: 1437-1440

[25] Malin SK, Bena J, Abood B et al. Attenuated improvements in adiponectin and fat loss characterize type 2 diabetes non-remission status after bariatric surgery. Diabetes Obes Metab 2014; 16: 1230-1238

[26] Khanna V, Malin SK, Bena J et al. Adults with long-duration type 2 diabetes have blunted glycemic and $\beta$-cell function improvements after bariatric surgery. Obesity 2015; 23: 523-526

[27] Lee W], Hur KY, Lakadawala M et al. Predicting success of metabolic surgery: age, body mass index, C-peptide, and duration score. Surg Obes Relat Dis 2013; 9: 379-384

[28] Still CD, Wood CG, Benotti P et al. A probability score for preoperative prediction of type 2 diabetes remission following RYGB surgery. Lancet Diabetes Endocrinol 2014; 2: 38-45

[29] Batterham RL, Cummings DE. Mechanisms of diabetes improvement following bariatric/metabolic surgery. Diabetes Care 2016; 39: 893 901

[30] Jackness C, Karmally W, Febres G et al. Very low-calorie diet mimics the early beneficial effect of Roux-en-Y gastric bypass on insulin sensitivity and $\beta$-cell function in type 2 diabetic patients. Diabetes 2013; 62: 3027-3032

[31] Lingvay I, Guth E, Islam A et al. Rapid improvement in diabetes after gastric bypass surgery: is it the diet or surgery? Diabetes Care 2013; 36 : $2741-2747$

[32] Steven S, Hollingsworth KG, Al-Mrabeh A et al. Very low-calorie diet and 6 months of weight stability in type 2 diabetes: pathophysiological changes in responders and nonresponders. Diabetes Care 2016; 39: $808-815$

[33] Khoo CM, Muehlbauer M], Stevens RD et al. Postprandial metabolite profiles reveal differential nutrient handling after bariatric surgery compared to matched caloric restriction. Ann Surg 2014; 259: 687- 693

[34] Steven S, Hollingsworth KG, Small PK et al. Weight loss decreases excess pancreatic triacylglycerol specifically in type 2 diabetes. Diabetes Care 2016; 39: $158-165$

[35] Sumithran P, Prendergast LA, Delbridge E et al. Long-term persistence of hormonal adaptations to weight loss. N Engl J Med 2011; 365: 1597 1604

[36] Salinari S, Bertuzzi A, Guidone C et al. Insulin sensitivity and secretion changes after gastric bypass in normotolerant and diabetic obese subjects. Ann Surg 2013; 257: 462-468
[37] Albers PH, Bojsen-Møller KN, Dirksen C et al. Enhanced insulin signaling in human skeletal muscle and adipose tissue following gastric bypass surgery. Am J Physiol Regul Integr Comp Physiol 2015; 309: R510 - R524

[38] Dadson P, Landini L, Helmiö M et al. Effect of bariatric surgery on adipose tissue glucose metabolism in different depots in patients with or without type 2 diabetes. Diabetes Care 2016; 39: 292 - 299

[39] Taylor R. Banting memorial lecture 2012 reversing the twin cycles of type 2 diabetes. Diabet Med 2013; 30: 267-275

[40] Lim EL, Hollingsworth KG, Aribisala BS et al. Reversal of type 2 diabetes: normalisation of beta cell function in association with decreased pancreas and liver triacylglycerol. Diabetologia 2011; 54: 2506-2514

[41] Hoffstedt J, Andersson DP, Hogling DE et al. Long-term protective changes in adipose tissue after gastric bypass. Diabetes Care 2017; 40: $77-84$

[42] Farey JE, Preda TC, Fisher OM et al. Effect of laparoscopic sleeve gastrectomy on fasting gastrointestinal, pancreatic, and adipose-derived hormones and on non-esterified fatty acids. Obes Surg 2017; 27: 399407

[43] Thomas F, Smith GC, Lu J et al. Differential acute impacts of sleeve gastrectomy, Roux-en-Y gastric bypass surgery and matched caloric restriction diet on insulin secretion, insulin effectiveness and non-esterified fatty acid levels among patients with type 2 diabetes. Obes Surg 2016; 26: $1924-1931$

[44] Lindegaard KK, Jorgensen NB, Just R et al. Effects of Roux-en-Y gastric bypass on fasting and postprandial inflammation-related parameters in obese subjects with normal glucose tolerance and in obese subjects with type 2 diabetes. Diabetol Metab Syndr 2015; 7: 12

[45] Lips MA, van Klinken JB, Pijl $\mathrm{H}$ et al. Weight loss induced by very low calorie diet is associated with a more beneficial systemic inflammatory profile than by Roux-en-Y gastric bypass. Metabolism 2016; 65: 16141620

[46] Kelly AS, Ryder JR, Marlatt KL et al. Changes in inflammation, oxidative stress and adipokines following bariatric surgery among adolescents with severe obesity. Int J Obes 2016; 40: 275-280

[47] Kratz M, Hagman DK, Kuzma JN et al. Improvements in glycemic control after gastric bypass occur despite persistent adipose tissue inflammation. Obesity 2016; 24: $1438-1445$

[48] Laferrère B, Teixeira J, McGinty J et al. Effect of weight loss by gastric bypass surgery versus hypocaloric diet on glucose and incretin levels in patients with type 2 diabetes. J Clin Endocrinol Metab 2008; 93: 24792485

[49] Bose M, Oliván B, Teixeira J et al. Do incretins play a role in the remission of type 2 diabetes after gastric bypass surgery: What are the evidence? Obes Surg 2009; 19: 217-229

[50] Manning S, Pucci A, Batterham RL. GLP-1: A mediator of the beneficial metabolic effects of bariatric surgery? Physiology (Bethesda) 2015; 30: $50-62$

[51] Goldfine AB, Mun EC, Devine E et al. Patients with neuroglycopenia after gastric bypass surgery have exaggerated incretin and insulin secretory responses to a mixed meal. J Clin Endocrinol Metab 2007; 92: 4678 4685

[52] Salehi M, Gastaldelli A, D'Alessio DA et al. Blockade of glucagon-like peptide 1 receptor corrects postprandial hypoglycemia after gastric bypass. Gastroenterology 2014; 146: 669-680.e2

[53] Dutia R, Brakoniecki K, Bunker $P$ et al. Limited recovery of $\beta$-cell function after gastric bypass despite clinical diabetes remission. Diabetes 2014; 63: $1214-1223$

[54] Chambers AP, Jessen L, Ryan KK et al. Weight-independent changes in blood glucose homeostasis after gastric bypass or vertical sleeve gastrectomy in rats. Gastroenterology 2011; 141: 950 - 958

[55] Service G], Thompson GB, Service FG et al. Hyperinsulinemic hypoglycemia with nesidioblastosis after gastric-bypass surgery. N Engl J Med 2005; 353: 249-254 
[56] Patti ME, McMahon G, Mun EC et al. Severe hypoglycaemia post-gastric bypass requiring partial pancreatectomy: evidence for inappropriate insulin secretion and pancreatic islet hyperplasia. Diabetologia 2005; 48: $2236-2240$

[57] Cummings DE. Gastric bypass and nesidioblastosis - Too much of a good thing for islets? N Engl J Med 2005; 353: 300 - 302

[58] Karamanakos SN, Vagenas K, Kalfarentzos F et al. Weight Loss, appetite suppression, and changes in fasting and postprandial ghrelin and peptide-YY levels after Roux-en-Y gastric bypass and sleeve gastrectomy: a prospective, double blind study. Ann Surg 2008; 247: 401-407

[59] Madsbad S, Holst J]. GLP-1 as a mediator in the remission of type 2 diabetes after gastric bypass and sleeve gastrectomy surgery. Diabetes 2014; 63: 3172-3174

[60] Holst J], Gribble F, Horowitz M et al. Roles of the gut in glucose homeostasis. Diabetes Care 2016; 39: 884-892

[61] Sloth B, Holst J], Flint A et al. Effects of PYY1-36 and PYY3-36 on appetite, energy intake, energy expenditure, glucose and fat metabolism in obese and lean subjects. Am J Physiol Endocrinol Metab 2007; 292 : E1062-E1068

[62] Manning S, Pucci A, Batterham RL. Roux-en-Y gastric bypass: effects on feeding behavior and underlying mechanisms. J Clin Invest 2015; 125 : 939-948

[63] Korner J, Inabnet W, Conwell IM et al. Differential effects of gastric bypass and banding on circulating gut hormone and leptin levels. Obesity 2006; 14: 1553-1561

[64] Meek CL, Lewis HB, Reimann F et al. The effect of bariatric surgery on gastrointestinal and pancreatic peptide hormones. Peptides 2016; 77: $28-37$

[65] Svane MS, Jørgensen NB, Bojsen-Møller KN et al. Peptide YY and glucagon-like peptide-1 contribute to decreased food intake after Roux-en-Y gastric bypass surgery. Int J Obes 2016; 40: 1699-1706

[66] Cummings DE, Weigle DS, Frayo RS et al. Plasma ghrelin levels after dietinduced weight loss or gastric bypass surgery. N Engl J Med 2002; 346: $1623-1630$

[67] Ochner CN, Gibson C, Shanik M et al. Changes in neurohormonal gut peptides following bariatric surgery. Int J Obes 2011; 35: 153-166

[68] Vigneshwaran B, Wahal A, Aggarwal S et al. Impact of sleeve gastrectomy on type 2 diabetes mellitus, gastric emptying time, glucagon-like peptide 1 (GLP-1), ghrelin and leptin in non-morbidly obese subjects with bmi 30-35.0 kg/m2: a prospective study. Obes Surg 2016; 26: $2817-2823$

[69] Nosso G, Griffo E, Cotugno M et al. Comparative effects of roux-en-y gastric bypass and sleeve gastrectomy on glucose homeostasis and incretin hormones in obese type 2 diabetic patients: a one-year prospective study. Horm Metab Res 2016; 48: 312-317

[70] Ramon JM, Salvans S, Crous $X$ et al. Effect of Roux-en-Y gastric bypass vs sleeve gastrectomy on glucose and gut hormones: a prospective randomised trial. J Gastrointest Surg 2012; 16: 1116-1122
[71] Scholtz S, Miras AD, Chhina N et al. Obese patients after gastric bypass surgery have lower brain-hedonic responses to food than after gastric banding. Gut 2014; 63: 891-902

[72] Sams VG, Blackledge C, Wijayatunga $N$ et al. Effect of bariatric surgery on systemic and adipose tissue inflammation. Surg Endosc 2016; 30: 3499-3504

[73] Bose M, Oliván B, Teixeira J et al. Do incretins play a role in the remission of type 2 diabetes after gastric bypass surgery: what are the evidence? Obes Surg 2009; 19: 217-229

[74] Rubino F, Forgione A, Cummings DE et al. The mechanism of diabetes control after gastrointestinal bypass surgery reveals a role of the proximal small intestine in the pathophysiology of type 2 diabetes. Ann Surg 2006; 244: $741-749$

[75] Saeidi N, Meoli L, Nestoridi E et al. Reprogramming of intestinal glucose metabolism and glycemic control in rats after gastric bypass. Science 2013; 341: $406-410$

[76] Salinari S, Debard C, Bertuzzi A et al. Jejunal proteins secreted by $\mathrm{db} / \mathrm{db}$ mice or insulin-resistant humans impair the insulin signaling and determine insulin resistance. PLOS ONE 2013; 8: e56258

[77] Salinari S, le Roux CW, Bertuzzi A et al. Duodenal-jejunal bypass and jejunectomy improve insulin sensitivity in Goto-Kakizaki diabetic rats without changes in incretins or insulin secretion. Diabetes 2014; 63: $1069-1078$

[78] Cavin JP, Couvelard A, Lebtahi R et al. Differences in alimentary glucose absorption and intestinal disposal of blood glucose after Roux-en-Y gastric bypass vs sleeve gastrectomy. Gastroenterology 2016; 150: 454464.e9

[79] Kir S, Beddow SA, Samuel VT et al. FGF19 as a postprandial, insulin-independent activator of hepatic protein and glycogen synthesis. Science 2011; 331: $1621-1624$

[80] Pournaras DJ, Glicksman C, Vincent RP et al. The role of bile after Rouxen-Y gastric bypass in promoting weight loss and improving glycaemic control. Endocrinology 2012; 153: 3613-3619

[81] Sachdev S, Wang Q, Billington C et al. FGF-19 and bile acids increase following Roux-en-Y gastric bypass but not after medical management in patients with type 2 diabetes. Obes Surg 2016; 5: 957-965

[82] Gerhard GS, Styer AM, Wood GC et al. A role for fibroblast growth factor 19 and bile acids in diabetes remission after Roux-en-Y gastric bypass. Diabetes Care 2013; 36: 1859-1864

[83] Albaugh VL, Flynn CR, Cai S et al. Early increases in bile acids post Rouxen-Y gastric bypass are driven by insulin-sensitizing, secondary bile acids. J Clin Endocrinol Metab 2015; 100: E1225-E1233

[84] Rubino F, Nathan DM, Eckel RH et al. Metabolic surgery in the treatment algorithm for type 2 diabetes: A joint statement by international diabetes organizations. Diabetes Care 2016; 39: 861-877 